\title{
Review of Some Numerical Methods for Solving Initial Value Problems for Ordinary Differential Equations
}

\author{
Fadugba Sunday Emmanuel ${ }^{1}$, Adebayo Kayode James ${ }^{1}$, Ogunyebi Segun Nathaniel ${ }^{1}$, \\ Okunlola Joseph Temitayo ${ }^{2}$ \\ ${ }^{1}$ Department of Mathematics, Ekti State University, Ado Ekiti, Nigeria \\ ${ }^{2}$ Department of Mathematical and Physical Sciences, Afe Babalola University, Ado Ekiti, Nigeria
}

\section{Email address:}

sunday.fadugba@eksu.edu.ng (F. S. Emmanuel)

\section{To cite this article:}

Fadugba Sunday Emmanuel, Adebayo Kayode James, Ogunyebi Segun Nathaniel, Okunlola Joseph Temitayo. Review of Some Numerical Methods for Solving Initial Value Problems for Ordinary Differential Equations. International Journal of Applied Mathematics and Theoretical Physics. Special Issue: Computational Mathematics. Vol. 6, No. 1, 2020, pp. 7-13. doi: 10.11648/j.ijamtp.20200601.12

Received: April 21, 2020; Accepted: April 30, 2020; Published: May 19, 2020

\begin{abstract}
Numerical analysis is a subject that is concerned with how to solve real life problems numerically. Numerical methods form an important part of solving differential equations emanated from real life situations, most especially in cases where there is no closed-form solution or difficult to obtain exact solutions. The main aim of this paper is to review some numerical methods for solving initial value problems of ordinary differential equations. The comparative study of the Third Order Convergence Numerical Method (FS), Adomian Decomposition Method (ADM) and Successive Approximation Method (SAM) in the context of the exact solution is presented. The methods will be compared in terms of convergence, accuracy and efficiency. Five illustrative examples/test problems were solved successfully. The results obtained show that the three methods are approximately the same in terms of accuracy and convergence in the case of first order linear ordinary differential equations. It is also observed that FS, ADM and SAM were found to be computationally efficient for the linear ordinary differential equations. In the case of the non-linear ordinary differential equations, SAM is found to be more accurate and converges faster to the exact solution than the FS and ADM. Hence, It is clearly seen that the ADM is found to be better than the FS and SAM in the case of non-linear differential equations in terms of computational efficiency.
\end{abstract}

Keywords: Accuracy, Adomian Decomposition Method, Convergence, Differential Equation, Efficiency, Initial Value Problem, Successive Approximation Method

\section{Introduction}

It is a known fact that several mathematical models emanating from the real and physical life situations cannot be solved explicitly, one has to compromise at numerical approximate solutions of the models achievable by various numerical techniques of different characteristics. Development of numerical methods for the solution of initial value problems in ordinary differential equations has attracted the attention of many researchers in recent years. Many authors have derived new numerical integration methods, giving better results than a few of the available ones in the literature such as: [1-11], just to mention a few.

The Adomian decomposition method (ADM) is an approximation method used for solving nonlinear differential equations, $[12,13]$. The proper use of the ADM has made it possible to obtain an analytic solution of a singular initialvalue problem when it is homogeneous or inhomogeneous $[14,15]$. Some of the merits of this method are that it converges fast to the exact solution [16], it requires less computational work than the other methods and it also has the ability to solve non-linear problems without linearizing. Some of the shortcomings of this method are that it gives a long series solution which must be cut short for it to be useful in practical application and the convergence rate for wider region is said to be slow [17]. A modified approach to the Adomian polynomials which converges a little faster than the original Adomian polynomials and is favourable for computer generation was introduced by [18]. A study for an effective modification of the ADM for solving second-order singular initial value problems was done and it was 
discovered that with few iterations used, the ADM is simple, easy to use and produces reliable results [19]. Successive approximation method is a means of solving initial value problems in ordinary differential equations numerically. It is useful when the solution of the differential equations cannot be obtained analytically. The method of successive approximations constitutes a so-called "algorithm or algorithmic process" for solving equations of a certain class in terms of a succession of elementary arithmetic operations. In this paper, the review of FS, ADM and SAM for the solution of linear and non-linear differential equations in the context of the exact solution is presented. The rest of the paper is organized as follows: Section Two presents the analysis of the methods. In Section Three, five illustrative examples were solved. Section Four presents the discussion of results and concluding remarks.

\section{Analysis of the Methods}

This section presents the analysis of the methods under consideration as follows:

\subsection{Analysis of the Third Order Convergence Numerical Method}

The third order convergence numerical method devised via the transcendental function of exponential type for the solution of the initial value problem in ordinary differential equation is given by [20].

$$
y_{n+1}=y_{n}+h f_{n}+\frac{h^{2}}{2}\left(f_{n}^{(1)}+f_{n}^{(2)}\right)+\left(1-e^{-h}-h\right) f_{n}^{(2)}
$$

\subsubsection{Local Truncation Error and Order of Accuracy of the Method}

Under localizing assumption, the local truncation error is obtained as

$$
\mathrm{LTE}=\frac{h^{4}}{24}\left(f^{(3)}-f_{n}^{(2)}\right)+\mathrm{O}\left(h^{5}\right)
$$

Thus, the leading term of the local truncation error involves $h^{4}$ confirms the third order accuracy of the numerical method given by (1) [20].

\subsubsection{Convergence Analysis of the Method}

The necessary and sufficient conditions for convergence are [21]

i. Consistency

ii. Stability

(i). Consistency Analysis of the Method

For a numerical method to be consistent, it is important for the truncation error to be zero when the step size, $h$, gets smaller and ultimately reaches to zero. Among many, one of the ways of analyzing consistency of a numerical technique is to check that whether (see [22]).

$$
\lim _{h \rightarrow 0} \frac{\text { LTE }}{h}=0
$$

From (2), we have that

$$
\lim _{h \rightarrow 0} \frac{h^{4}}{24}\left(f^{(3)}-f_{n}^{(2)}\right) / h=\lim _{h \rightarrow 0} \frac{h^{3}}{24}\left(f^{(3)}-f_{n}^{(2)}\right)=0(4)
$$

Equation (3) has been established. From (4), it is observed that the method has consistency property.

(ii). Stability Analysis of the Method

For stability analysis of the scheme (1), one of the popular ways is to apply it to the test problem of the form

$$
y^{\prime}=-\lambda y, y(0)=1
$$

with the theoretical solution of the form

$$
y(x)=e^{-\lambda x}, \lambda>0
$$

Where $\lambda$ is, in general, a complex constant.

For the integration interval $\left[x_{n}, x_{n+1}\right]$, where $h=x_{n+1}-$ $x_{n}$; the exact solution at the point $x=x_{n+1}$ is

$$
y\left(x_{n+1}\right)=e^{-\lambda x_{n+1}}=e^{-\lambda x_{n}} \cdot e^{-\lambda h}=y\left(x_{n}\right) \cdot e^{-\lambda h}
$$

where $h$ is defined as $\left(x_{n+1}-x_{n}=h\right)$. The numerical approximation obtained using the proposed technique gives

$$
y_{n+1}=y_{n}\left(1-\lambda h+\frac{\lambda^{2} h^{2}}{2 !}-\frac{\lambda^{3} h^{3}}{3 !}\right)
$$

Let

$$
B=1-\lambda h+\frac{\lambda^{2} h^{2}}{2 !}-\frac{\lambda^{3} h^{3}}{3 !}
$$

Then

$$
y_{n+1}=\mathrm{B} y_{n}
$$

Comparing (7) and (10), this shows that the factor B is merely an approximation for the factor $e^{-\lambda h}$ in the exact solution. Truly, the factor B is the four-term approximation for the Maclaurin's series for $e^{-\lambda h}$ for small $\lambda \mathrm{h}$. The error growth factor $\mathrm{B}$ can be controlled by $\|\mathrm{B}\|<1$, so that the errors may not magnify. Thus, the stability of the third order convergence method requires that

$$
\left\|1-\lambda h+\frac{\lambda^{2} h^{2}}{2 !}-\frac{\lambda^{3} h^{3}}{3 !}\right\|<1
$$

Setting $z=\lambda h$, then $(11)$ becomes

$$
\left\|1-\lambda z+\frac{z^{2}}{2 !}-\frac{z^{3}}{3 !}\right\|<1
$$

\subsection{Analysis of the Adomian Decomposition Method}

Consider the following equation

$$
L y+R y+N y=h(x)
$$

where $N$ is a non-linear operator, $L$ is the highest order derivative and $R$ is a linear differential operator. Taking the $L^{-1}$ of both sides of (13), one obtains;

$$
y=L^{-1} h(x)-L^{-1} N y-L^{-1} R y
$$

Since $L$ is assumed to be invertible, in general one can 
define $L^{-1}$ for $L=\frac{d^{n}}{d x^{n}}$ as the $\mathrm{n}$-fold definite integration with interval $[0, x]$. Thus, from (14), one gets

$$
y=C+D x+L^{-1} h(x)-L^{-1} N y-L^{-1} R y
$$

where $A$ and $B$ are constants of integration. ADM assumes that the unknown function $y$ can be expressed by an infinite series of the form

$$
y=\sum_{n=0}^{\infty} y_{n}(x)
$$

where the components $y_{n}(x)$ will be determined recursively. Moreover, the method defines the nonlinear term by the Adomian's polynomials. ADM also assumes that the nonlinear operator $N(y)$ can be decomposed by an infinite series of polynomials given by

$$
N(y)=\sum_{n=0}^{\infty} A_{n}
$$

with

$$
A_{n}=\frac{1}{n !} \frac{d^{n}}{d \lambda^{n}} N\left(\sum_{j=0}^{\infty} \lambda^{j} y_{j}\right)_{\lambda=0}, n=0,1,2, \ldots
$$

where (13) represents the Adomian polynomials of $y_{0}, y_{1}, y_{2}, \ldots, y_{n}$.

By means of (16), (17) and (18), one gets

$$
\Theta=\sum_{n=0}^{\infty} y_{n}(x)
$$

with

$$
\Theta=C+D x+L^{-1} h(x)-L^{-1} R\left(\sum_{n=0}^{\infty} y_{n}(x)\right)-L^{-1}\left(\sum_{n=0}^{\infty} A_{n}\right)
$$

The recursive formula is given by

$$
\left.\begin{array}{l}
y_{0}=g(x) \\
y_{n+1}=-L^{-1} R y_{n}-L^{-1} A_{n}
\end{array}\right\}
$$

Using (21), the solution of $y$ can be obtained as follows

$$
y=\lim _{n \rightarrow \infty} \sum_{j=0}^{n} y_{j}
$$

The order and the rate of convergence of the Adomian decomposition method are summarized below [23].

Definition 1

$$
\mathrm{y}_{0}(\mathrm{x})=y_{0}, y_{1}(x)=y_{0}+\int_{x_{0}}^{x} f\left(x, y_{0}(t)\right) d t, y_{1}(x)=y_{0}+\int_{x_{0}}^{x} f\left(x, y_{0}(t)\right) d t, \ldots
$$

The following result gives the existence and uniqueness theorem for the successive approximation method [26]
For every $n \in N \cup\{0\}$, we define the rate of convergence as

$$
\alpha_{n}=\left\{\begin{array}{c}
\frac{\left\|y_{n+1}\right\|}{\left\|y_{n}\right\|},\left\|y_{n}\right\| \neq 0 \\
0,\left\|y_{n}\right\|=0
\end{array}\right.
$$

Theorem 1

Let $N$ be an operator from a Hilbert space $H$ into $H$ and y be exact solution of $y=N(y)+f$. Then the sum, $\sum_{j=0}^{n-1} \mathrm{yn}$, which is obtained from (19) converges to y such that $0 \leq \alpha<1,\left\|y_{n+1}\right\| \leq \alpha\left\|y_{n}\right\|, \forall n \in N U\{0\}$.

Remark 1

a) The expression in Theorem 1 that is $\sum_{j=0}^{n-1} y_{n}$ converges to an exact solution $\mathrm{y}$, when $0 \leq \alpha_{n}<1, n=1,2,3, \ldots$

b) A detailed proof of the convergence of ADM using the entire series property can be found in [24]

\subsection{Analysis of the Successive Approximation Method}

Here, the successive approximation method will be analyzed as follows [25].

Consider the initial value problem of first order ordinary differential equation of the form

$$
y^{\prime}(x)=\mathrm{f}(x, y), y\left(x_{0}\right)=y_{0}
$$

Integral representation of (23) yields

$$
\int_{x_{0}}^{x} y^{\prime}(x) d x=\int_{x_{0}}^{x} f(x, y(x)) d x
$$

Solving (24) further one gets

$$
y(x)-y\left(x_{0}\right)=\int_{x_{0}}^{x} f(x, y(x)) d x
$$

Substituting the initial condition $y\left(x_{o}\right)=y_{o}$ into (25), yields

$$
y(x)=y_{0}+\int_{x_{0}}^{x} f(x, y(x)) d x .
$$

Changing the variable of integration to $t$ instead of $x$ in (26) gives,

$$
y(x)=y_{0}+\int_{x_{0}}^{x} f(x, y(t)) d t
$$

Equation (27) is used to generate a successive approximation of solution to the initial value problem (23) which is of the form:

$$
y_{n}(x)=y_{0}+\int_{x_{0}}^{x} f\left(x, y_{n-1}(t)\right) d t, n=1,2,3, \ldots
$$

Hence, the successive approximations are obtained as: 
region $D$. Let $M$ be a constant such that

$$
|f(x, y)|<M \text { in } D
$$

Also, let $f(x, y)$ in $D$ satisfies the Lipschitz condition:

$$
\left|f\left(x, y_{1}\right)-f\left(x, y_{2}\right)\right| \leq \mathrm{A}\left|y_{1}-y_{2}\right|
$$

where $\mathrm{A}$ is independent of $x, y_{1}, y_{2}$.

Suppose there exists a rectangle $R$ defined by

$$
\left|x-x_{0}\right| \leq h,\left|y-y_{0}\right| \leq k
$$

such that $M h<k$ where $R \subseteq D$. Then for $\left|x-x_{0}\right| \leq h$, $y^{\prime}=f(x, y)$ has a unique solution $y=y(x)$ for which $y\left(x_{0}\right)=y_{0}$.

\section{Illustrative Examples}

It is usually necessary to demonstrate the suitability and applicability of the methods. In this course, the algorithms of the methods have been successfully translated into the MATLAB programming language and implemented on some initial value problems. The performance of the methods has been checked by comparing their accuracy and efficiency with the exact solution. The efficiency was determined from the number of iterations counts and number of function evaluations per step while the accuracy is determined by the size of the discretization error estimated from the difference between the exact solution and the numerical approximations.

Problem 1

Consider the initial value problem of first order linear ordinary differential equation

$$
y^{\prime}-2 x y=0, \quad y(0)=1
$$

The exact solution of (33) is obtained as

$$
y(x)=\exp \left(x^{2}\right)
$$

The comparative results analyses of the FS, ADM and SAM in the context of the theoretical solution are displayed in Figure 1 below. The errors incurred in the FS, ADM and SAM are shown in Figure 2 below.

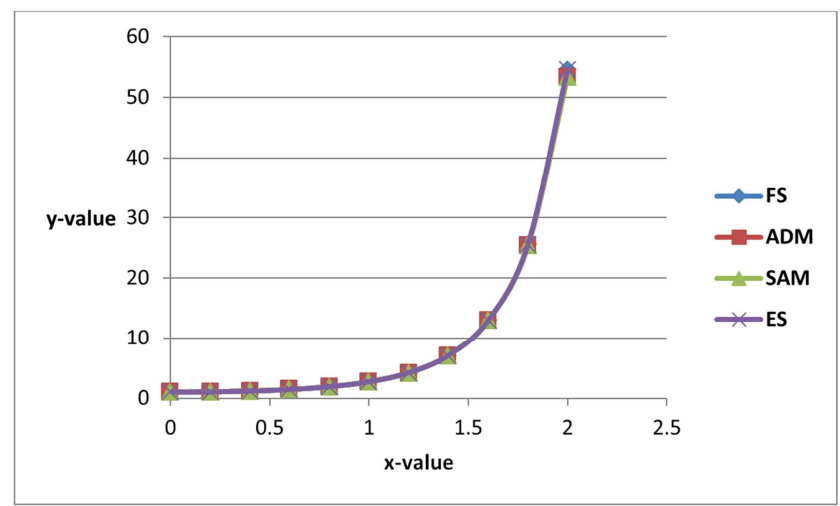

Figure 1. The comparative results analyses of the ADM and SAM in the context of the Exact Solution (ES) for different values of $x$.

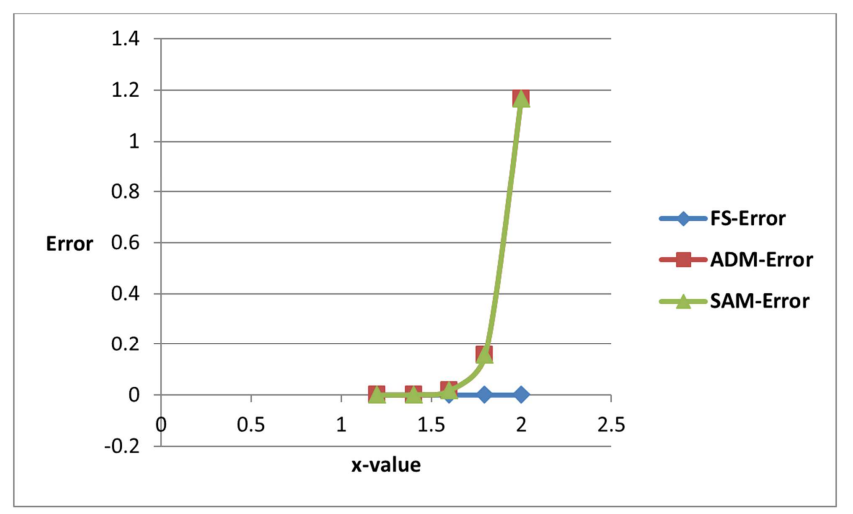

Figure 2. The errors incurred in the FS, ADM and SAM for different values of $x$.

Problem 2

Consider the initial value problem of first order linear ordinary differential equation

$$
y^{\prime}-y=0, \quad y(0)=1
$$

The exact solution of (35) is obtained as

$$
y(x)=\exp (x)
$$

The comparative results analyses of the ADM and SAM in the context of the exact solution are displayed in Figure 3 below.

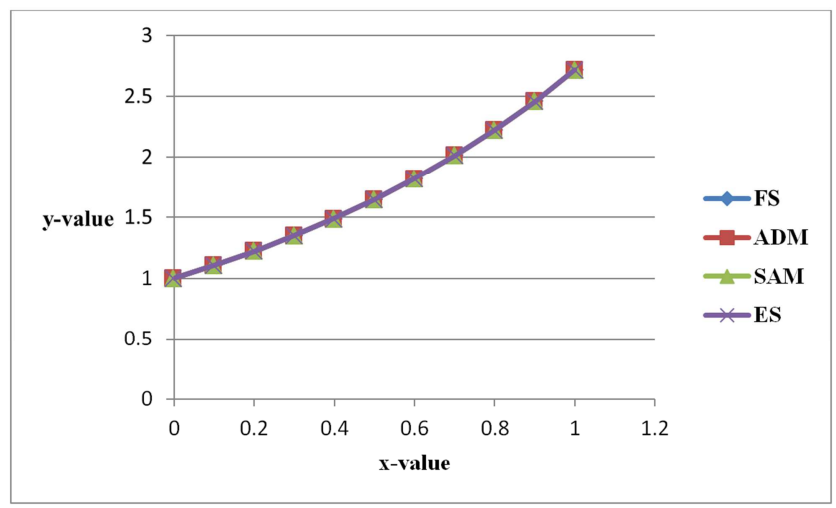

Figure 3. The comparative results analyses of the FS, ADM and SAM in the context of the Exact Solution (ES) for different values of $x$.

Problem 3

Consider the initial value problem of first order non-linear ordinary differential equation

$$
y^{\prime}=1-y^{2}, \quad y(0)=0
$$

The exact solution of (37) is obtained as

$$
y(x)=\frac{1-\exp (-2 x)}{1+\exp (-2 x)}
$$

The comparative results analyses of the FS, ADM and SAM in the context of the exact solution are displayed in 
Figure 4 below. The errors incurred in the FS, ADM and SAM are shown in Figure 5 below.

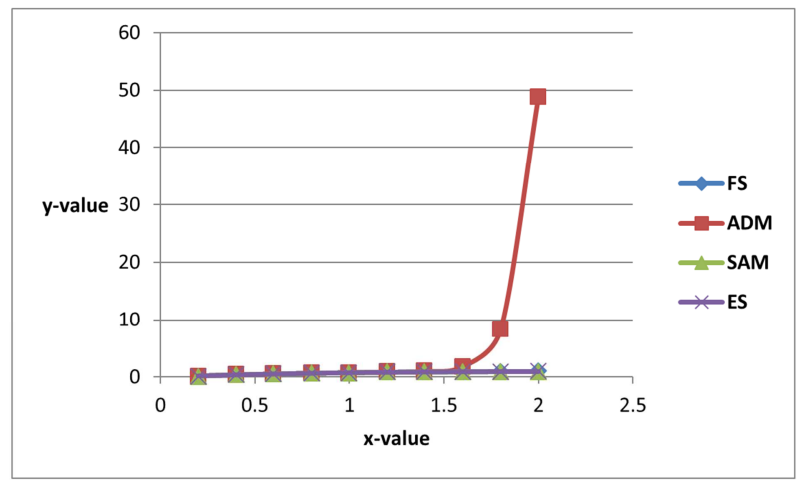

Figure 4. The comparative results analyses of the FS, ADM and SAM in the context of the Exact Solution (ES) for different values of $x$.

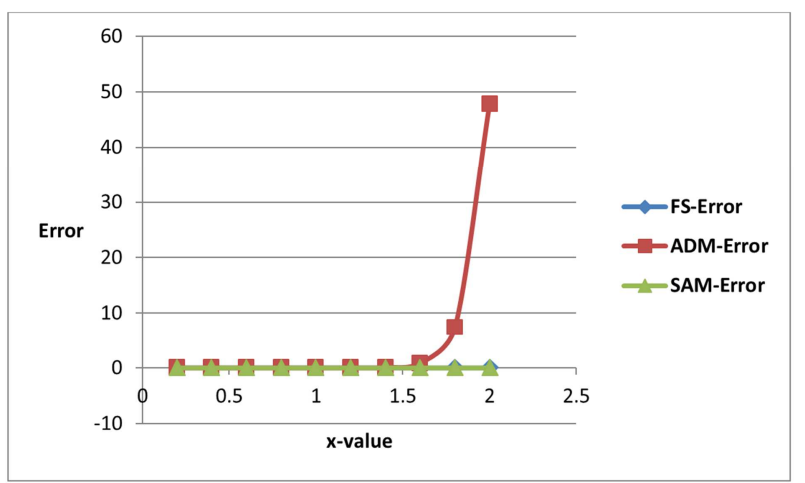

Figure 5. The errors incurred in the FS, ADM and SAM for different values of $x$.

Problem 4

Consider the initial value problem of third order non-linear ordinary differential equation

$$
y^{\prime \prime \prime}=-y y^{\prime \prime}+\left(y^{\prime}\right)^{2}+3 y^{\prime}, \quad y(0)=0, y^{\prime}(0)=-2
$$

The exact solution of (39) is obtained as

$$
y(x)=\frac{1}{2}(1-\exp (-2 x))
$$

The comparative results analyses of the FS, ADM and SAM in the context of the exact solution are displayed in Figure 6 below. The errors incurred in the FS, ADM and SAM are shown in Figure 7 below.

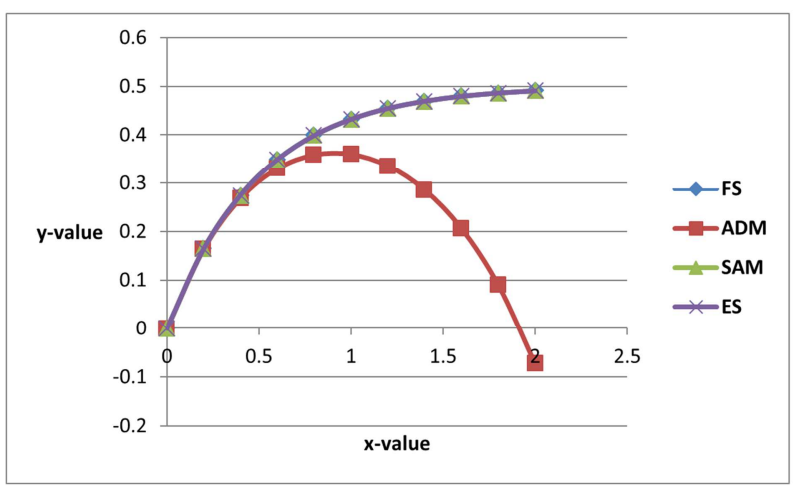

Figure 6. The comparative results analyses of the FS, ADM and SAM in the context of the Exact Solution (ES) for different values of $x$.

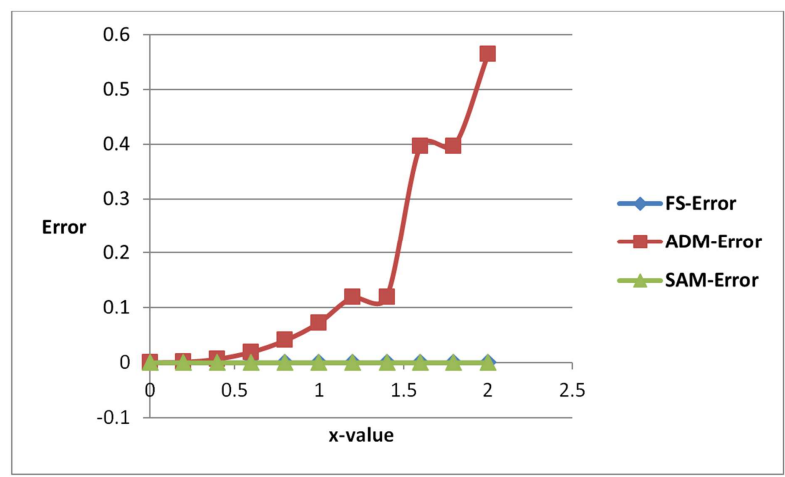

Figure 7. The errors incurred in the FS, ADM and SAM for different values of $x$.

Problem 5

Consider the initial value problem of first order non-linear ordinary differential equation

$$
y^{\prime}-y^{2}=0 \quad y(0)=1
$$

The exact solution of (41) is obtained as

$$
y(x)=\frac{1}{1-x}
$$

The comparative results analyses of the FS, ADM and SAM in the context of the exact solution are displayed in Figure 8 below. The errors incurred in the FS, ADM and SAM are shown in Figure 9 below.

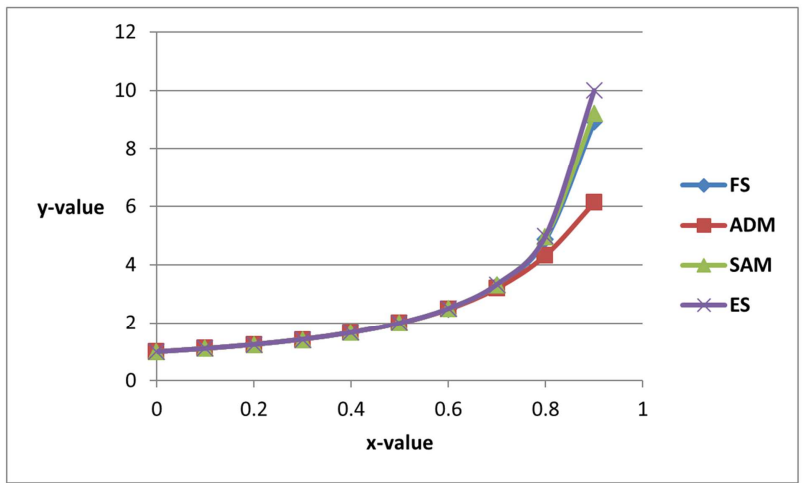

Figure 8. The comparative results analyses of the FS, ADM and SAM in the context of the Exact Solution (ES) for different values of $x$. 


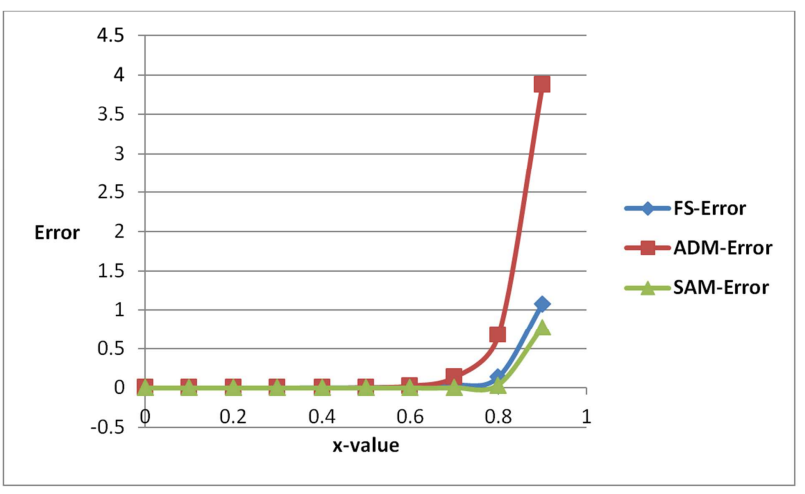

Figure 9. The error incurred in the FS, ADM and SAM for different values of $x$.

\section{Discussion of Results and Concluding Remarks}

In this paper, the review of some numerical methods, namely third order convergence numerical method known as "FS" devised via a transcendental interpolating function of exponential type, ADM and SAM for the solution of linear and non-linear differential equations is presented. The comparative results analyses of FS, ADM and SAM in the context of the exact solution are also presented. The performance of the FS, ADM and SAM is measured based on the accuracy, efficiency and convergence. Five illustrative examples were considered. The results generated via the three methods show that they are good tools for the solution of initial value problems of first order linear ordinary differential equations; see Figures 1 and 3. It is also observed that FS is more accurate than ADM and SAM as shown in Figure 2. It is observed from Figures 4, 6 and 8 that FS and SAM performed better than ADM in terms of accuracy in the case of the non-linear differential equations. It is also observed from Figures 5, 7 and 9 that SAM converges faster to the exact solution than the FS and ADM. In terms of efficiency, ADM has performed excellently since the run time of ADM is less than that of FS and SAM in the case of nonlinear ordinary differential equations. Some extensions and modifications of the methodology can be explored by further research. A natural extension is the applications of FS, ADM and SAM for the solution of some special initial value problems of higher order differential equations emanating from the real life problems with the point of singularity.

\section{References}

[1] N. Ahmad, S. Charan, and V. P. Singh, Study of numerical accuracy of Runge-Kutta second, third and fourth order method, 2015.

[2] Y. Ansari, A. Shaikh, and S. Qureshi. Error bounds for a numerical scheme with reduced slope evaluations, J. Appl. Environ. Biol. Sci., 8 (7), 2018.

[3] J. C. Butcher, Numerical methods for ordinary differential equations, John Wiley \& Sons, 2016.
[4] M. E. Davis, Numerical methods and modeling for chemical engineers, Courier Corporation, 2013.

[5] S. E. Fadugba, Numerical technique via interpolating function for solving second order ordinary differential equations, Journal of Mathematics and Statistics, 1 (2): 1-6, 2019.

[6] S. E. Fadugba and A. O. Ajayi, Comparative study of a new scheme and some existing methods for the solution of initial value problems in ordinary differential equations, International Journal of Engineering and Future Technology, 14: 47-56, 2017.

[7] S. Fadugba and B. Falodun, Development of a new one-step scheme for the solution of initial value problem (IVP) in ordinary differential equations, International Journal of Theoretical and Applied Mathematics, 3: 58-63, 2017.

[8] S. E. Fadugba and J. T. Okunlola, Performance measure of a new one-step numerical technique via interpolating function for the solution of initial value problem of first order differential equation, World Scientific News, 90: 77-87, 2017.

[9] S. E. Fadugba and T. E. Olaosebikan, Comparative study of a class of one-step methods for the numerical solution of some initial value problems in ordinary differential equations, Research Journal of Mathematics and Computer Science, 2: 111, 2018, DOI: $10.28933 /$ rjmcs-2017-12-1801.

[10] R. B. Ogunrinde and S. E. Fadugba, Development of the New Scheme for the solution of Initial Value Problems in Ordinary Differential Equations, International Organization of Scientific Research Journal of Mathematics (IOSRJM), 2: 24-29, 2012.

[11] S. E. Fadugba and S. Qureshi, Convergent numerical method using transcendental function of exponential type to solve continuous dynamical systems, Punjab University Journal of Mathematics, 51: 45-56. 2019.

[12] G. Adomian, A review of the decomposition in applied mathematics, Mathematical Analysis and Applications, 135: 501-544, 1988.

[13] G. Adomian, A review of the decomposition method and some recent results for nonlinear equation. Mathematical Computational Model, 13 (7), 1992.

[14] A. M. Wazwaz, A reliable modification of Adomian decomposition method. Applied Mathematics and Computation, 102: 77-86, 1999.

[15] A. M. Wazwaz, A new method for solving singular initial value problems in the second order ordinary differential equations, Applied Mathematics and Computation, 128: 45$57,2002$.

[16] A. H. M. Abdelrazec, Adomian decomposition method convergence analysis and numerical approximations, M. Sc., McMaster University, 2008.

[17] S. M. Holmquist, An examination of the effectiveness of the Adomian decomposition method in fluid dynamic applications, Ph. D., University of Central Florida, 2007.

[18] G. Adomian and R. Rach, Modified Adomian polynomials, Mathematical Computational Model., 24 (11): 39-46, 1996.

[19] Y. Q. Hasan and L. M. Zhu, Modified Adomian decomposition method for singular initial value problems in the second order ordinary differential equations. Surveys in Mathematics and its Applications, 3: 183-193, 2008. 
[20] S. E. Fadugba and J. O. Idowu, Analysis of the properties of a third order convergence numerical method derived via transcendental function of exponential form, International Journal of Applied Mathematics and Theoretical Physics, 5: 97-103, 2019.

[21] J. D. Lambert, Numerical methods for ordinary differential systems: the initial value problem, John Wiley \& Sons, Inc., New York, 1991.

[22] S. Qureshi and S. E. Fadugba, Convergence of a numerical technique via interpolating function to approximate physical dynamical systems, Journal of Advanced Physics, 7: 446-450, 2018.

[23] S. S. Motsa and S. Shateyi, New analytic solution to the laneemden equation of index 2, Mathematical problems in Engineering, pp. 1-20, 2012.
[24] Kent Nagle, Edward B. Saff, and Arthur David Snider, Fundamentals of differential equations and boundary value problems, Fourth edition, Pearson Addison Wesley, 2004.

[25] Y. Cherruault, G. Saccomandi, and B. Some, New results for convergence of Adomian method applied to integral equations, Mathematical Computational Modelling., 16: 8593, 1992.

[26] Y. O. Fashomi, On the comparison between Picard's iteration method and Adomain decomposition method in solving nonlinear differential equations, Structured Masters, African Institute of Mathematical Sciences (AIMS), 2013. 